\title{
Heart Rate Variability and Perceptual Well-Being in Triathlon - A Case Report
}

\author{
Bernhard Grässler* and Anita Hökelmann \\ Department of Sport Science, Otto von Guericke University, Germany
}

*Corresponding author: Bernhard Grässler, Department of Sport Science, Faculty of Humanities, Otto von Guericke University, Magdeburg, Germany.

To Cite This Article: Bernhard Grässler, Anita Hökelmann. Heart Rate Variability and Perceptual Well-Being in Triathlon - A Case Report. Am J Biomed Sci \& Res. 2021 - 12(5). AJBSR.MS.ID.001791. DOI: 10.34297/AJBSR.2021.12.001791.

Received: 眥 April 23, 2021; Published: 跚 April 30, 2021

\begin{abstract}
Fluctuations in the intervals between adjacent heartbeats are described as heart rate variability (HRV). In professional sports, HRV is a popular tool to evaluate regeneration and prevent the state of overtraining. HRV measurements could also be used as a simple, non-invasive method to evaluate personal well-being of athletes and prescribe training. However, more understanding in the association between HRV and psychophysiological factors are necessary. Therefore, the aim of this study was to examine the relationship between resting HRV and personal well-being in two triathletes. HRV was recorded at least four times per week for a period of five weeks. Daily personal well-being was assessed via questionnaire. A significant positive correlation between RMSSD and well-being was found for one athlete, while there was no correlation for the other athlete. Further research with bigger sample sizes is needed and should include collection of performance data and other physiological measures.
\end{abstract}

Keywords: Heart rate variability; Autonomic function; Triathlon; Well-Being; Physical stress

Abbreviations: ANS: Autonomic Nervous System; ECG: Electrocardiography; HRV: Heart Rate Variability; RMSSD: Root of the Mean Square of the Differences of successive NN intervals

\section{Introduction}

Heart rate variability (HRV) describes the variations in the time between adjacent heart beats and is the result of the complex interaction between sympathetic and parasympathetic activity [1]. As HRV reflects the balance of the autonomic nervous system (ANS) and cardiovascular stability [1], it is regarded as an indicator of neurocardiac function [2]. Relatively high HRV reflects good cardiovascular health and adaptability of the ANS [3]. Furthermore, HRV is a non-invasive marker for the assessment of the risk for cardiovascular diseases [4]. Meanwhile, HRV measurement is a popular method to monitor the training process and has the potential to identify a stress-regeneration imbalance [5]. Although HRV measurements are beneficial in exercise training prescription, more understanding of the relationship between HRV and perceptual well-being is necessary for an efficient HRVguided training process [6]. Therefore, the purpose of the present study was to determine the relationship between resting HRV and perceptual well-being of two triathletes. According to literature [7], we hypothesized a positive correlation between HRV measures and well-being.

\section{Materials and Methods}

Data of two (S1 and S2) athletes will be presented here. Over a period of five weeks, the athletes conducted by themselves HRV measurements with the Omegawave system (Espoo, Finland) at resting state. The measurements were conducted in the morning and according to the recommended procedure of the company. The athletes recorded HRV at least four times per week. The HRV parameter RMSSD (root of the mean square of the differences of successive NN intervals), reflecting vagal activity, which is relevant for psychophysiology, and less affected by respiration compared to frequency-domain parameters, was used for HRV analysis [8]. During the study period, athletes completed a psychological questionnaire every day. The questionnaire assessed muscle soreness, stress, mood, fatigue, and sleep quality on a five-point scale with scores from 1 (very bad) to 5 (very good) [9]. As data 
were not normal distributed, Spearman correlation was used to correlate RMSSD and the sum of the five scores of the questionnaire (well-being) for each day when HRV was recorded.

\section{Results and Discussion}

Athlete S1 conducted $26 \mathrm{HRV}$ recordings, while athlete S2 conducted $21 \mathrm{HRV}$ recordings. Spearman correlation showed a significant positive correlation between RMSSD and well-being for $\mathrm{S} 1(\mathrm{p}=.006 ; \mathrm{r}=.528)$. However, there was no correlation for $\mathrm{S} 2 \mathrm{p}$ $=.406 ; \mathrm{r}=-.191$ ). The changes in HRV of athlete $\mathrm{S} 1$ reflected the changes in his well-being, so that increases in HRV were associated with better well-being and vice versa. However, no such correlation was detected in athlete $\mathrm{S} 2$. One explanation for this contradictory finding could be the smaller number of HRV recordings in athlete S2. Furthermore, the questionnaire is subjective and reflects in a simple way only the current well-being of the athlete, whereas HRV reflects the accumulated response of the last few training sessions on autonomic functioning. Finally, HRV measurements were not controlled by an investigator and Omegawave does not provide any artefact correction. HRV recordings with artefacts were discarded only by visual inspection.

\section{Conclusion}

In summary, a positive relationship between HRV data and perceptual well-being over a period of five weeks was found in athlete S1 but not for athlete S2. More studies with bigger sample sizes, performance data, and ECG measurements for HRV analysis are needed to assess the importance of HRV as a marker of wellbeing in athletes and as a tool to prescribe training.

\section{Acknowledgements}

The authors have nothing to acknowledge.

\section{Conflict of Interest}

The authors declare that the research was conducted in the absence of any commercial or financial relationships that could be construed as a potential conflict of interest.

\section{References}

1. Shaffer F, McCraty R, Zerr CL (2014) A healthy heart is not a metronome: An integrative review of the heart's anatomy and heart rate variability. Front Psychol 30(5): 1040.

2. McCraty R, Shaffer F (2015) Heart rate variability: New perspectives on physiological mechanisms, assessment of self-regulatory capacity, and health risk. Glob Adv Health Med 4(1): 46-61.

3. Jung, W, Jang, K-I, Lee S-H (2019) Heart and Brain Interaction of Psychiatric Illness: A Review Focused on Heart Rate Variability, Cognitive Function, and Quantitative Electroencephalography. Clin Psychopharmacol Neurosci 17(4): 459-474.

4. Cripps TR, Malik M, Farrell TG, Camm AJ (1991) Prognostic value of reduced heart rate variability after myocardial infarction: Clinical evaluation of a new analysis method. Br Heart J 65(1): 14-19.

5. Buchheit M (2014) Monitoring training status with HR measures: Do all roads lead to Rome? Front Physiol 5(73).

6. Kiviniemi Antti M, Hautala Arto J, Kinnunen H, Nissilä J, Virtanen P, et al. (2010) Daily exercise prescription on the basis of HR variability among men and women. Med Sci Sports Exerc 42(7): 1355-1363.

7. Le Meur Y, Pichon A, Schaal K, Schmitt L, Louis, J, et al. (2013) Evidence of parasympathetic hyperactivity in functionally overreached athletes. Med Sci Sports Exerc 45(11): 2061-2071.

8. Laborde S, Mosley E, Thayer JF (2017) Heart rate variability and cardiac vagal tone in psychophysiological research - Recommendations for experiment planning, data analysis, and data reporting. Front Psychol 8: 213.

9. Hooper SL, Mackinnon LT, Howard A, Gordon RD, Bachmann AW (1995) Markers for monitoring overtraining and recovery. Med Sci Sports Exerc 27(1): 106-112. 